\title{
Diffusion Anomaly in an Associating Lattice Gas Model
}

\author{
Marcia M. Szortyka \\ Instituto de Física, Universidade Federal do Rio Grande do Sul, Caixa Postal 15051, \\ 91501-970, Porto Alegre, RS, Brazil \\ Marcia C. Barbosa $\S$ \\ Instituto de Física, Universidade Federal do Rio Grande do Sul, Caixa Postal 15051, \\ 91501-970, Porto Alegre, RS, Brazil
}

\begin{abstract}
.
We investigate the relation between thermodynamic and dynamic properties of an associating lattice gas (ALG) model. The ALG combines a two dimensional lattice gas with particles interacting through a soft core potential and orientational degrees of freedom. From the competition between the directional attractive forces and the soft core potential results two liquid phases, double criticality and density anomaly. We study the mobility of the molecules in this model by calculating the diffusion constant at a constant temperature, $D$. We show that $D$ has a maximum at a density $\rho_{\max }$ and a minimum at a density $\rho_{\min }<\rho_{\max }$. Between these densities the diffusivity differs from the one expected for normal liquids. We also show that in the pressure-temperature phase-diagram the line of extrema in diffusivity is close to the liquid-liquid critical point and it is inside the temperature of maximum density (TMD) line.
\end{abstract}

$\S$ To whom correspondence should be addressed (marcia.barbosa@ufrgs.br) 


\section{Introduction}

Water is anomalous substance in many respects. Most liquids contract upon cooling. This is not the case of water, a liquid where the specific volume at ambient pressure starts to increase when cooled below $T=4^{\circ} \mathrm{C}$ [1]. Besides, in a certain range of pressures, also exhibits an anomalous increase of compressibility and specific heat upon cooling [2]- 4].

Far less known are its dynamics anomalies: while for most materials diffusivity decreases with increasing pressure, liquid water has an opposite behavior in a large region of the phase diagram [5]-[13]. The increase of diffusivity of water as the pressure is increased is related to the competition between the local ordered tetrahedral structure of the first neighbours and the distortions of the structure of the first and second neighbours. In the region of the phase diagram where this ordered structure is dominant, increasing pressure implies breaking first neighbours hydrogen bonds what allow for interstitial second neighbours to be in a closer approach. The interactions are thus weakened and therefore, although the system is more dense, it has a larger mobility. In this sense, a good model for water and tetrahedral liquids should not only exhibit thermodynamic but also dynamic anomalies. In SPC/E water, the region of the pressure-temperature $(\mathrm{p}-\mathrm{T})$ phase diagram where the density anomaly appears is contained within the region of the $\mathrm{p}-\mathrm{T}$ phase diagram where anomalies in the diffusivity are present 9, 10].

It was proposed a few years ago that these anomalies are related to a second critical point between two liquid phases, a low density liquid (LDL) and a high density liquid (HDL) [14]. This critical point was discovered by computer simulations. This work suggests that this critical point is located at the supercooled region beyond the line of homogeneous nucleation and thus cannot be experimentally measured. Even if this limitation, this hypothesis has been supported by indirect experimental results [15, 16].

Water, however, is not an isolated case. There are also other examples of

tetrahedrally bonded molecular liquids such as phosphorus [17, 18, and amorphous silica [19] that also are good candidates for having two liquid phases. Moreover, other materials such as liquid metals [20] and graphite [21] also exhibit thermodynamic anomalies. Unfortunately, a closed theory giving the relation between the form of the interaction potential and the presence of the anomalies is still missing.

It was observed that water has both diffusion and density anomalous behavior and that they are located in the same region of the $p-T$ phase diagram. It is reasonable to think that the dynamics and thermodynamics are deeply related. In this case establishing the connection between the anomalous behavior of the diffusion constant and the density anomaly is a fundamental step towards understanding the source of the anomalies. In order to test this assumption, the dynamics of a number of models in which density anomaly is present were studied [22, 23]. Netz et al [22] studied a system of molecules interacting by a purely repulsive ramp-like discretized potential consisting of a $n$ number of steps of equal size. If $n$ is small, the region on the $p$ - $T$ phase diagram in which the density anomaly is present encloses the region in which the diffusion anomaly 
exists. If $n$ is large, the potential becomes equivalent to a smooth ramp [23] and the region $p-T$ phase diagram in which the diffusion anomaly is present encloses the region in which the density anomaly exists. This behavior resembles the one expected for water. Unfortunately these two models do not exhibit a second critical point (or even a first critical point) because the interaction potential in both cases is purely repulsive. Therefore no connection with criticality and dynamic and thermodynamic anomalies could be made.

The difficulty in including the attractive interactions in these approaches is that within continuous potentials attractive terms usually move the density anomaly to the metastable region of the $p$ - $T$ phase diagram. Moreover continuous potentials usually lead to crystallization at the region where the critical point would be expected. The analysis of the presence of both the two liquid phases and the critical point becomes indirect.

In order to circumvent these difficulties, our model system is the a lattice gas with ice variables [24] which allows for a low density ordered structure [25, 26]. Competition between the filling up of the lattice and the formation of an open four-bonded orientational structure is naturally introduced in terms of the ice bonding variables and no ad hoc introduction of density or bond strength variations is needed. Our approach bares some resemblance to that of some continuous models [27, 28, 29, which, however, lack entropy related to hydrogen distribution on bonds. Also, the reduction of phase-space imposed by the lattice allows construction of the full phase diagram from simulations, not always possible for continuous models [27. The associating lattice gas model (ALG) [25, 26] is able to exhibit for a convenient set of parameters both density anomalies and the two liquid phases. In the framework of the ALG we address two questions: (i) is the presence of diffusion anomaly related to the presence of density anomaly? (ii) if so, what is the hierarchy between the two anomalies and the presence of a second critical point? We show that the two anomalies are located in the same region of the $p-T$ phase diagram, close to the second critical point and that the region on the $p-T$ phase diagram in which the density anomaly is present encloses the region in which the diffusion anomaly exists.

\section{The Model}

We consider the ALG that is a two dimensional lattice gas model on a triangular lattice as introduced by Henriques and Barbosa [25, 26]. The sites that can be empty or occupied. Besides the occupational variable, $\sigma_{i}$, which assumes the value $\sigma_{i}=0$ if the site is empty, or $\sigma_{i}=1$ if the site is full, there are other six bond variables, $\tau_{i}^{i j}$, representing the possibility of hydrogen bond formation between the neighbours sites. Four bonding variables are the ice bonding arms, two donor with $\tau_{i}^{i j}=1$ and two acceptor with $\tau_{i}^{i j}=-1$. The other two arms are taken as inert, with $\tau_{i}^{i j}=0$, and are always opposite to each other, as illustrated in Fig. 1. Because there are no restrictions on the bonding arms positions, the particles are allowed to be in eighteen different states. 


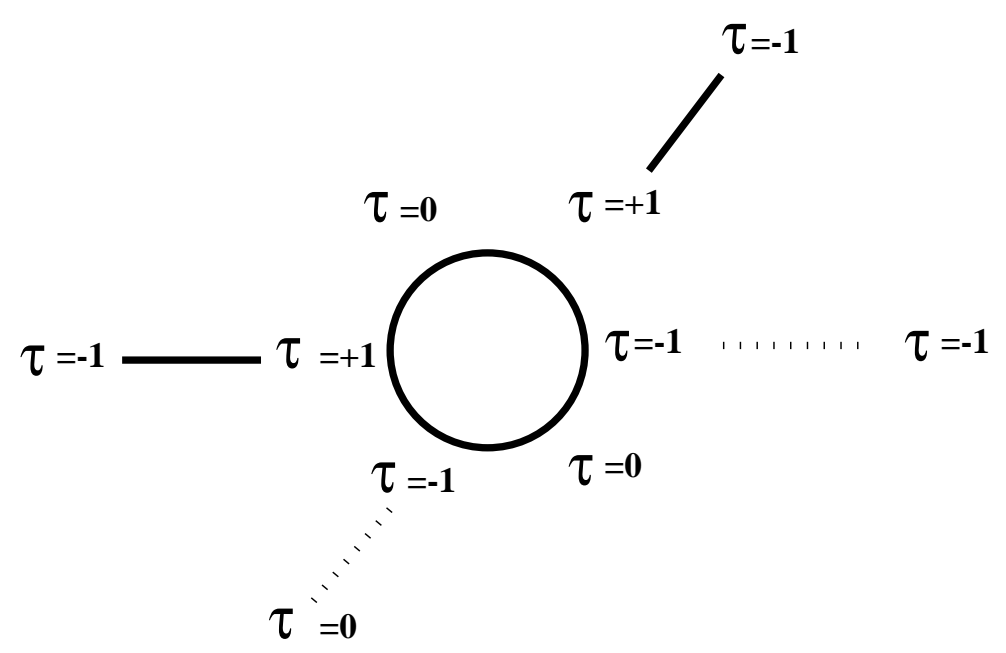

Figure 1. Illustration of the model. Each site $i$ can be empty or occupied and has six variables, $\tau_{i}^{k}$, one for each arm. If $\tau_{i}^{k}=0$ no bond is formed in spite of the configuration of the arm of the neighbour site, if $\tau_{i}^{l}= \pm 1$ and the arm of the neighbour site has $\tau_{j}^{l}=\mp 1$, a bond is formed.

The Hamiltonian of the model has two kinds of interactions: one isotropic attractive, like van der Waals, and a repulsive orientational hydrogen bonding. The energy of the system is given by

$$
E=(-v+2 u) \sum_{\langle i, j\rangle} \sigma_{i} \sigma_{j}+u \sum_{\langle i, j\rangle} \sigma_{i} \sigma_{j} \sum_{k=1}^{6} \sum_{l}^{*}\left[\left(1-\tau_{i}^{k} \tau_{j}^{l}\right) \tau_{i}^{k} \tau_{j}^{l}\right],
$$

where $-v+2 u$ is the van der Waals interaction, $-2 u$ is the hydrogen bonds interaction, $\sigma_{i}=0,1$ are occupation variables, $\tau_{i}^{k}=0, \pm 1$ represent the bonding arms variables as illustrated in Fig. 1, the summation over $k$ is over the arms of the site we are considering and the summation over $l$ is over the six neighbours arms that especific point to the site arm. Two neighbour sites $i$ and $j$ form an hydrogen bond if the product between their pointing arms is equal to -1 , in other words, we need $\tau_{i}^{k} \tau_{j}^{l}=-1$. In spite of each molecule has six neighbours, only four hydrogen bonds are allowed for site. For each pair of occupied sites that form a hydrogen bond, an energy $-v$ is attributed, while for non-bonding pairs of occupied sites, the energy is $-v+2 u$.

The phase diagram of the reduced chemical potential, $\bar{\mu}=\mu / v$, versus reduced temperature, $\bar{T}=k_{B} T / v$ reproduced in Fig. 2 was originally obtained by Henriques and Barbosa [25] using Monte Carlo simulations for a triangular lattice with $L^{2}=100$ lattice sites. At low temperatures and chemical potentials, the system is in the gas phase. As the the chemical is increased at constant temperature, there is a first-order phase transition between the gas to a low density liquid phase $(L D L)$. As the chemical potential is increased even further, there is another first-order transition between the LDL phase a high density liquid phase $(H D L)$. The two transition end at critical points.

The reduced pressure, $\bar{P}=P / v$ versus reduced temperature phase diagram was also obtained by Henriques and Barbosa [25] and it is illustrated in Fig. 3. In the 


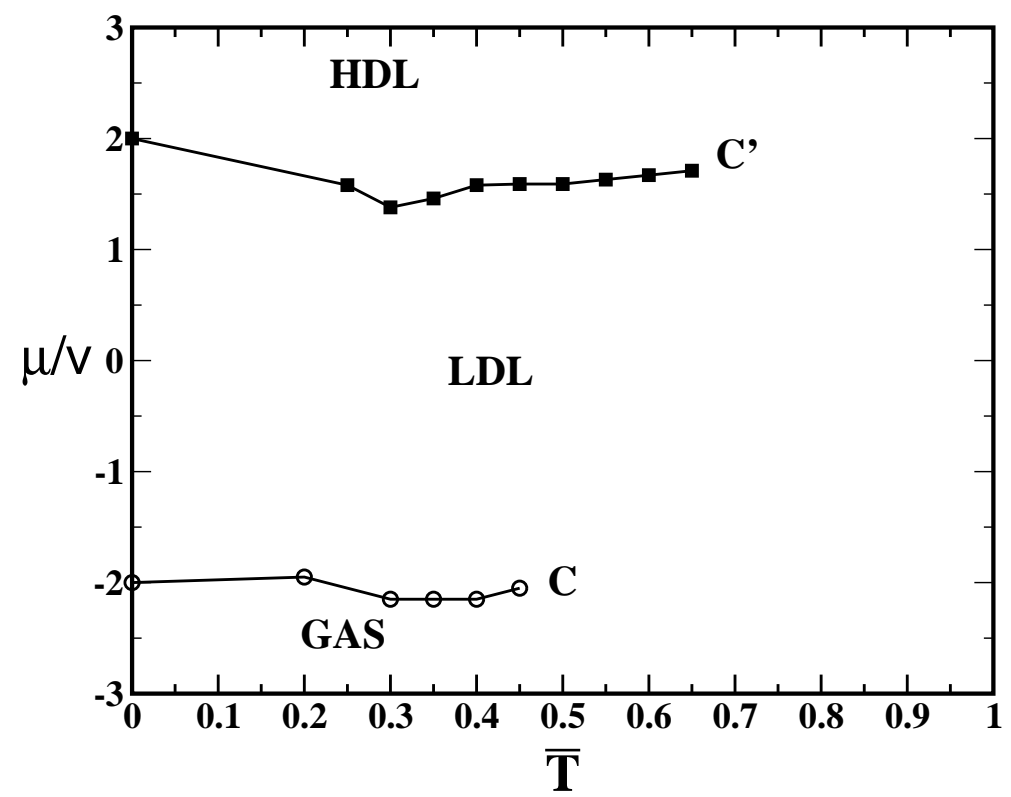

Figure 2. Phase diagram showing reduced chemical vs. reduced temperature for $u / v=1$. The filled symbols represent the LDL-HDL coexistence line. The empty symbols indicate the gas-LDL coexistence line. The coexistence at zero temperature at $\bar{\mu}=-2$ and $\bar{\mu}=2$ are exact.

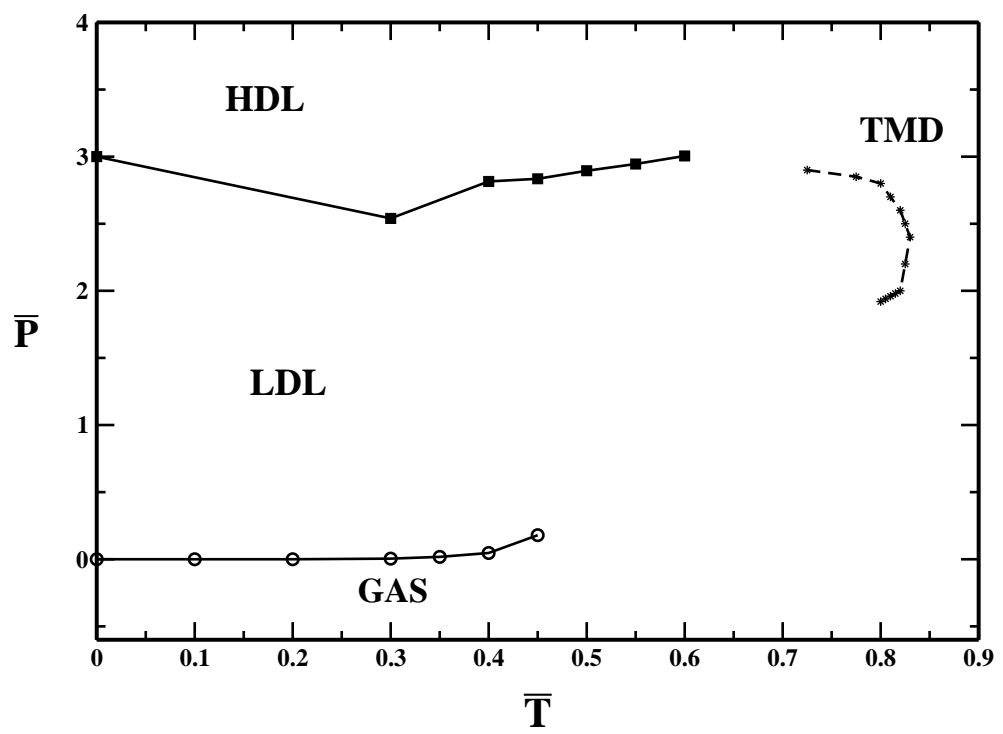

Figure 3. Phase diagram showing reduced pressure vs. reduced temperature for $u / v=1$. The filled symbols represent the LDL-HDL coexistence line. The empty symbols indicate the gas-LDL coexistence line. The coexistence at zero temperature at $p / v=3$ and $p / v=0$ are exact. The dashed line is the TMD. 
$\bar{P}-\bar{T}$ plane, close to the $L D L-H D L$ critical point, there is a region of temperature of maximum density $(T M D)$ for a given pressure. Surprisingly the location of the continuous transitions, the critical points and the density anomaly are not very sensitive to the systems size. Some test runs were done for $L^{2}=400,2500,4900$. The difference between the critical points obtained by applying finite size scaling to those runs and the result for $L^{2}=100$ is quite small. So, for simplicity all the detailed study of the model properties and the full phase diagrams was undertaken for an $L^{2}=100$ lattice sites.

This simple model exhibits two liquid phases, two critical points and density anomaly similar to the ones present in the SPC/E model for water and in other potential models for water. In the next section we will investigate if it also has diffusion anomaly and how this anomaly is related to the $T M D$.

\section{Diffusion}

For studying the mobility, we have performed Monte Carlo simulations of a system of $n$ particles interacting as specified by the Hamiltonian Eq.(1) in a triangular lattice with $L^{2}=100$ lattice sites. The procedure for computing the diffusion coefficient goes as follows. The system is equilibrated at a fixed chemical potential and temperature. In equilibrium this system has $n$ particles. Starting from this equilibrium configuration at a time $t=0$, each one of these $n$ particles is allowed to move to an empty neighbour site randomly chosen. The move is accepted if the total energy of the system is reduced by the move, otherwise it is accepted with a probability $\exp \left(\Delta E / k_{B} T\right)$ where $\Delta E$ is the difference between the energy of the system after and before the move. After repeating this procedure $n t$ times, the mean square displacement per particle at a time $t$ is computed by

$$
\left\langle\Delta r(t)^{2}\right\rangle=\left\langle(\mathbf{r}(t)-\mathbf{r}(0))^{2}\right\rangle,
$$

where $\mathbf{r}(0)$ is the particle position at the initial time and $\mathbf{r}(t)$ is the particle position at a time $t$. In Eq. (4), the average is taken over all particles and over different initial configurations. The diffusion coefficient is then obtained from the relation

$$
D=\lim _{t \rightarrow \infty} \frac{\left\langle\Delta r(t)^{2}\right\rangle}{4 t} .
$$

Since the time is measured in Monte Carlo time steps and the distance in number of lattice distance, a dimensionless diffusion coefficient is defined as

$$
\bar{D}=\lim _{t \rightarrow \infty} \frac{\left\langle\Delta \bar{r}(t)^{2}\right\rangle}{4 \bar{t}} .
$$

where $\bar{r}=r / a$ and $a$ is the distance between two neighbour sites and $\bar{t}=t / t_{M C}$ is the time in Monte Carlo steps.

In order to find if our system exhibits diffusion anomalies, we have analysed how $\bar{D}$ varies with the number density $\rho=n / L^{2}$ for a fixed temperature. For each temperature and chemical potential, 200 samples were obtained and an average over samples was made. 


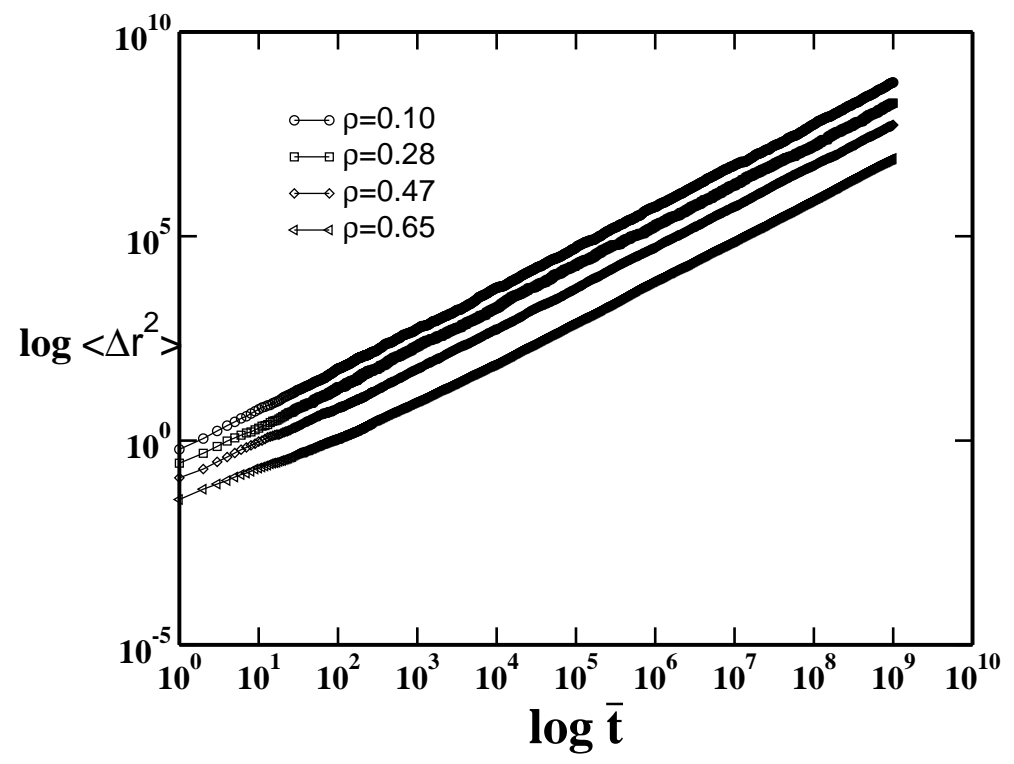

Figure 4. Mean square displacement vs. reduced time in logarithm scale for $\bar{T}=0.8$ and densities from top to bottom $\rho=0.1,0.28,0.47,0.65$.

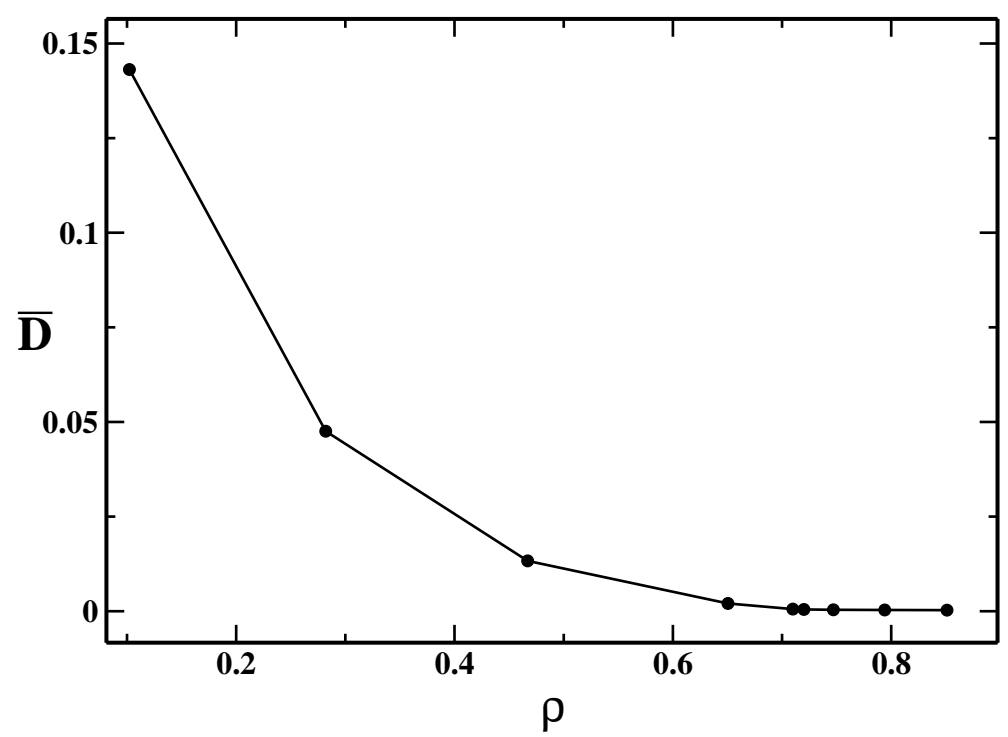

Figure 5. Reduced Diffusion coefficient vs. density for $\bar{T}=0.8 . \bar{D}$ increases with the reductions of the density as in a normal liquid.

At low chemical potentials and $\bar{T}=0.8, \rho$ is small and the mean square displacement has only the diffusive regime as illustrated in Fig. 4. As the chemical potential is increased, $\rho$ also increases and the ballistic regime appears. Using the ballistic regime, the mean square displacement was then computed for densities ranging from $\rho=0.1$ to $\rho=0.9$ ( not illustrated in Fig. 4 for clarity). From this data together with Eq. (44), we have obtained the reduced diffusion coefficient, $\bar{D}$, versus density, $\rho$, shown in Fig. 5. In this case, $\bar{D}$ increases with the decrease of the density what 


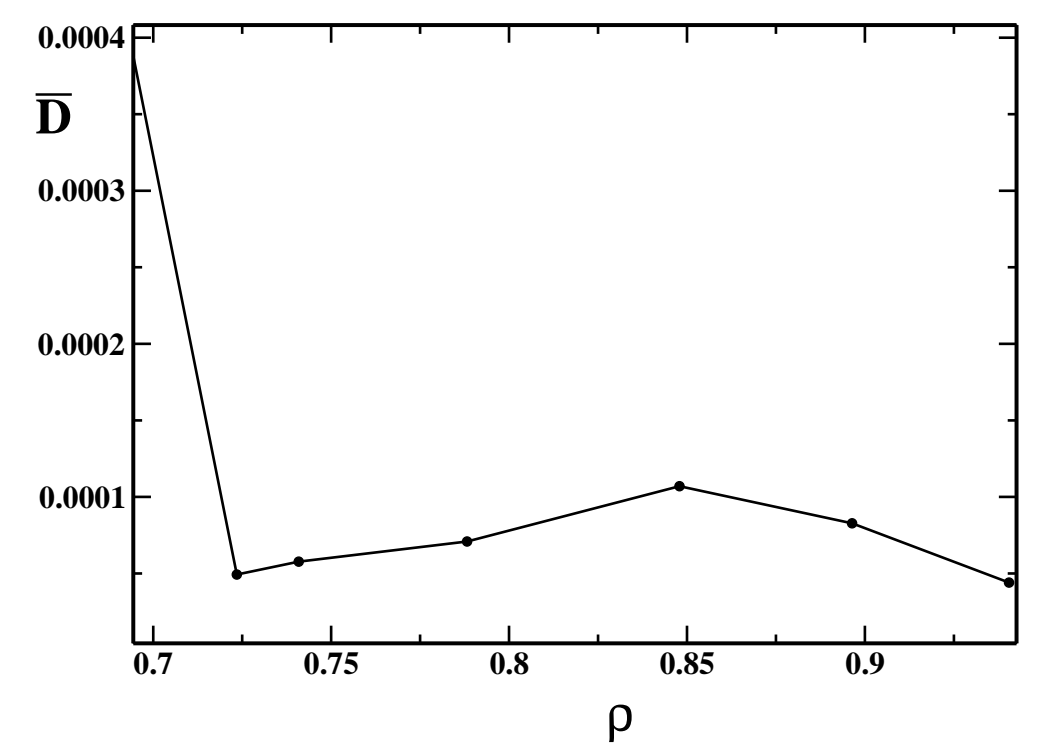

Figure 6. Reduced Diffusion coefficient vs. density for $\bar{T}=0.75$.

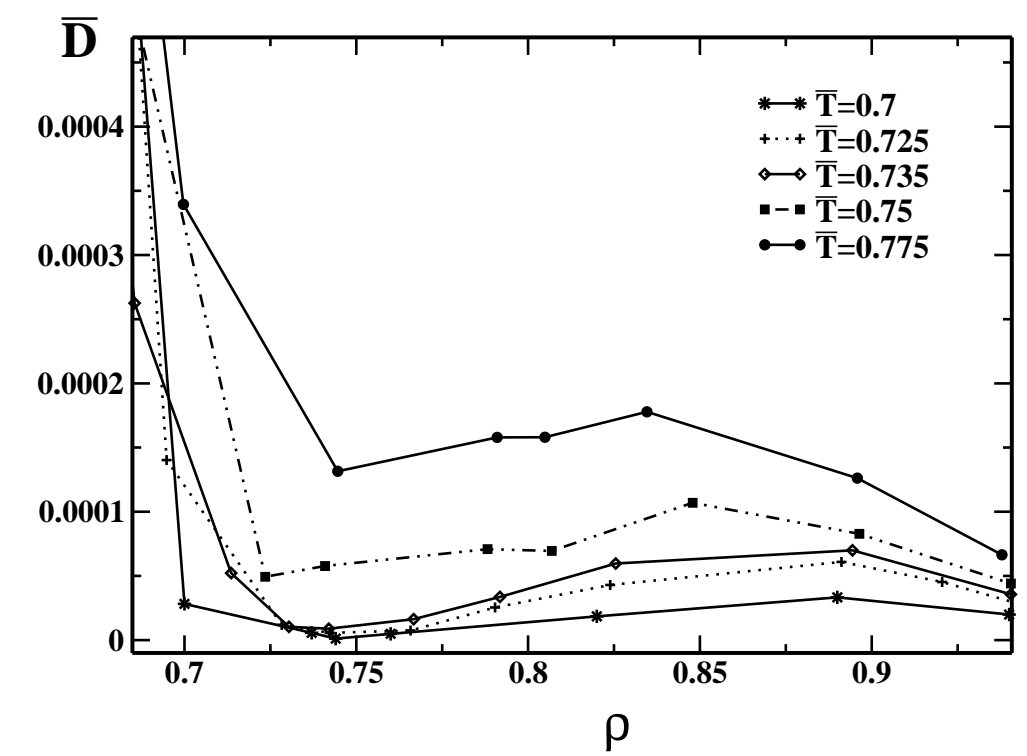

Figure 7. Reduced Diffusion coefficient vs. density for $T=$ $0.7,0.725,0.735,0.75,0.775$.

represents the behavior expected for normal liquids. Simulations for higher temperatures are consistent with this result.

However, this is not the case at lower temperatures. For instance, the mean square displacement for $\bar{T}=0.75$ exhibits a peculiar behavior different from the one observed in Fig. 4. At the diffusive regime, for a certain density range, the slope of $\left\langle(\Delta r)^{2}\right\rangle$ instead of increasing with the decrease of density, decreases with it. Consequently the isochores cross each other. The reduced diffusion coefficient, $\bar{D}$ ( shown in Fig. 6) at very low densities decreases as $\rho$ increases like in a normal liquid. However, as the density is 


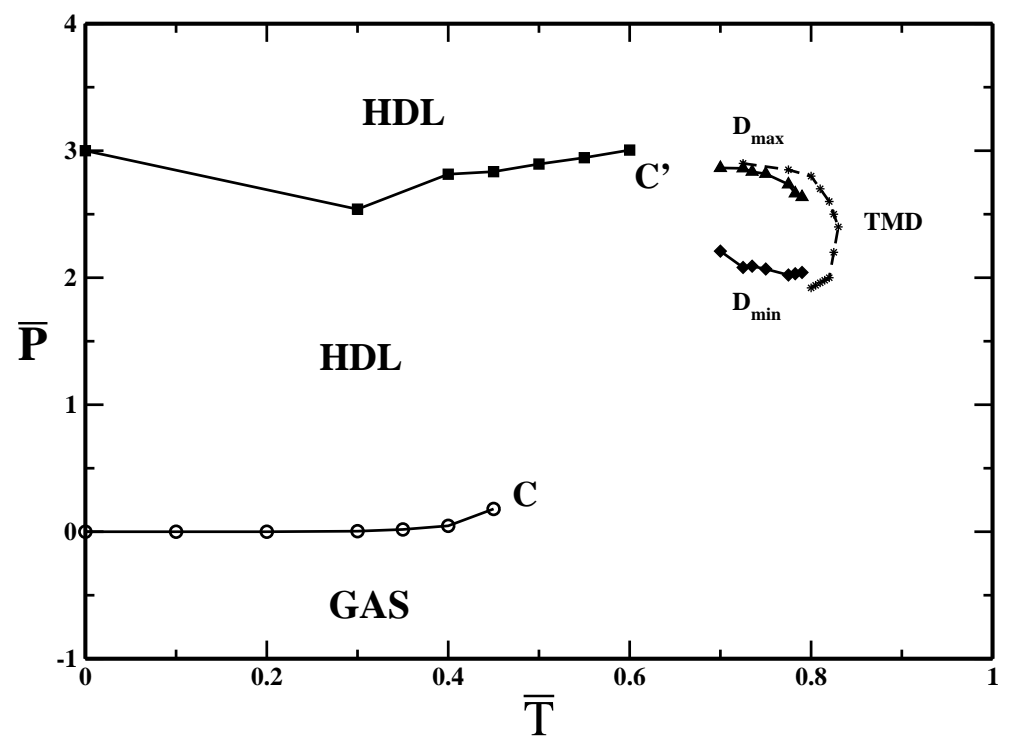

Figure 8. Reduced pressure vs. reduced temperature phase diagram showing the the two liquid phase, two critical points, the density anomaly and the diffusion anomaly regions.

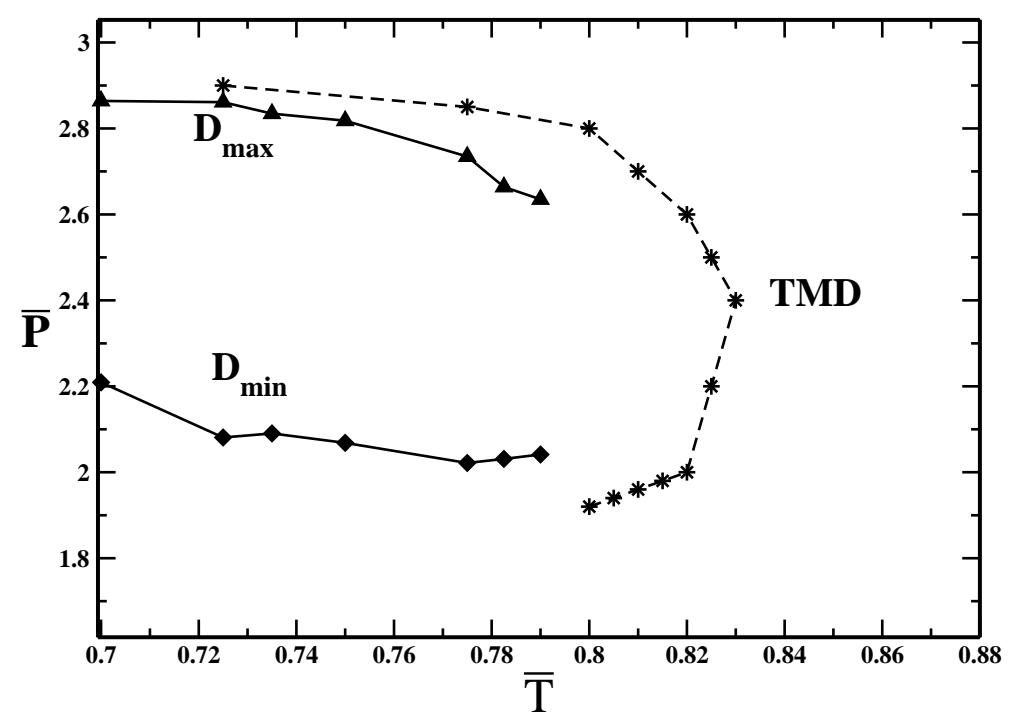

Figure 9. Zoom of the reduced pressure vs. reduced temperature phase diagram showing the density anomaly and diffusion anomaly regions.

increased $\bar{D}$ has a minimum at $\rho_{D m i n}$, and increases with the increase of density from $\rho_{D_{\min }}<\rho<\rho_{D_{\max }}$. Increasing the density above $\rho_{D \max }, \bar{D}$ decreases as in a normal liquid. Therefore, there is a region of densities $\rho_{D \max }>\rho>\rho_{D \min }$ where the diffusion coefficient is anomalous, increasing with density. This behavior is similar to the diffusion anomaly present in SPC/E water and ramp and shoulder models. A diffusion anomaly in the ALG model is observed in the range of temperatures $0.8>\bar{T}>0.6$ illustrated in Fig. 7. 
The region in the $p-T$ plane where there is an anomalous behavior in the diffusion is bounded by $\left(T_{D \min }, P_{D \min }\right)$ and $\left(T_{D \max }, P_{D \max }\right)$ and their location is shown in Fig. 8. The region of diffusion anomalies $\left(T_{D \max }, P_{D \max }\right)$ and $\left(T_{D \min }, P_{D \min }\right)$ lies inside the region of density anomalies ( for detail see fig. 9) what differs from the behavior observed in $\mathrm{SPC} / \mathrm{E}$ water [10] but coincides with the behavior shown for non smooth ramp-like potentials [22].

\section{Conclusions}

In this paper we have addressed two questions: (i) is the presence of diffusion anomaly related to the presence of density anomaly? (ii) if so, what is the hierarchy between the two anomalies and the presence of a second critical point?

For tracking the answer of these two questions we have investigated the behavior of the diffusion coefficient in the associating lattice gas model. This simple model is suitable for addressing our two questions since it exhibits two liquid phases and a line of density anomalies (TMD) [25, 26].

Using Monte Carlo simulations we computed the the mean square displacement with time. From the slope of this curve at the diffusive regime, the diffusion coefficient was derived. This procedure was implement for different temperatures and densities. We found that for high temperatures, particles in our model system diffuse as a normal liquid. At low temperatures, however, the diffusion coefficient has an interval of densities $\rho_{D_{\min }}<\rho<\rho_{D_{\max }}$ where $\bar{D}$ increases with the increase of density. Therefore, the presence of a density anomaly seems to be associated with the presence of diffusion anomaly, confirming observations made in other models [23, 30] and in water [10, 12, 13. This seems to indicate that as the particles gain more energy by being close together, this gain facilitates the mobility.

For addressing the second question, we have located in the $p-T$ phase diagram the pressure of the density of maximum diffusivity and the pressure of the density of

minimum diffusivity. Since for each temperature there is one $\rho_{D_{\min }}$ and there is one $\rho_{D_{\max }}$, the diffusivity extrema is composed of two lines at the $p-T$ phase-diagram. By comparing the $T M D$ with the lines of diffusivity extrema, we found that the region in the $p$ - $T$ plane of diffusion anomaly is located inside the region of the density anomaly and that both are in the vicinity of the second critical point. This suggests that in models where anomalies are present, the second critical point might arises if a attractive term in the potential would be included. The hierarchy between the anomalies resembles the one observed in the purely repulsive ramp-like discretized potential [22]. The link between the two models is the presence of two competing interaction distances and the non smooth transition between them. The first ingredient seems to be the one that defines the presence of the anomalies, while the second might govern the hierarchy between them [22] 23] 30]. Similar behavior should be expected in other models where the density anomaly is also present [31]- 35$]$ 


\section{Acknowledgements}

We thank to Jeferson Arenzon for having helped us with the computational method of diffusion in Monte Carlo. We thank and the Brazilian science agencies CNPq, Capes, Finep and Fapergs for financial support.

[1] R. Waller, Essays of Natural Experiments, Johnson Reprint corporation, New York, 1964.

[2] F. X. Prielmeir, E. W. Lang, Rl. Speedy, H. -D. Lüdemann, Phys. Rev. Lett. 59, 1128 (1987).

[3] F. X. Prielmeier, E. W. Lang, R. J. Speedy, H. -D. Lüdemann, B. Bunsenges, Phys. Chem. 92, 1111 (1988).

[4] L. Haar, J. S. Gallagher, G. S. Kell, NBS/NRC Steam Tables. Thermodynamic and Transport Properties and Computer Programs for Vapor and Liquid States of Water in SI Units, Hemisphere Publishing Co., Washington DC, 1984, pp271-276.

[5] F. W. Starr, F. Sciortino and H. E. Stanley, Phys. Rev. E 60, 6757 (1999); F. W. Starr, S. T. Harrington, F. Sciortino and H. E. Stanley, Phys. Rev. Lett. 82, 3629 (1999).

[6] P. Gallo, F. Sciortino, P. Tartaglia and S. -H. Chen, Phys. Rev. Lett. 76, 2730 (1996); F. Sciortino, P. Gallo, P. Tartaglia and S. -H. Chen, Phys. Rev. E 54, 6331 (1996); S. -H. Chen, F. Sciortino and P. Tartaglia, ibid. 56, 4231 (1997); F. Sciortino, L. Fabbian, S. -H. Chen and P. Tartaglia ibid. 56, 5397 (1997).

[7] S. Harrington, P. H. Poole, F. Sciortino and H. E. Stanley, J. Chem. Phys. 107, 7443 (1997).

[8] F. Sciortino, A. Geiger and H. E. Stanley, Nature (London) 354, 218 (1991); J. Chem Phys. 96, 3857 (1992).

[9] J. R. Errington and P. G. Debenedetti, Nature (London) 409, 318 (2001).

[10] P. A. Netz, F. W. Starr, H. E. Stanley and M. C. Barbosa, J. Chem. Phys. 115, 344 (2001).

[11] P. A. Netz, F. W. Starr, M. C. Barbosa and H. E. Stanley, Physica A 314, 470 (2002).

[12] H. E. Stanley, M. C. Barbosa, S. Mossa, P. A. Netz, F. Sciortino, F. W. Starr and M. Yamada, Physica A 315, 281 (2002).

[13] P. A. Netz, F.W. Starr, M. C. Barbosa and H. E. Stanley, J. Mol. Liquids 101, 159 (2002).

[14] P.H. Poole, F. Sciortino, U. Essmann, and H. E. Stanley, Nature extbf360, 324 (1992); Phys. Rev. E 48, 3799 (1993); F. Sciortino, P.H. Poole, U. Essmann, and H.E. Stanley, Ibid 55, 727 (1997); S. Harrington, R. Zhang, P.H. Poole, F. Sciortino, and H.E. Stanley, Phys. Rev. Lett. 78, 2409 (1997).

[15] O. Mishima, H. E. Stanley, Nature 396, 329 (1998).

[16] R.J. Speedy and C.A. Angell, J Chem Phys 65, 851 (1976).

[17] Y. Katayama, T. Mizutani, W. Utsumi, O. Shimomura, M. Yamakata and K. Funakoshi, Nature 403, 170 (2000).

[18] G. Monaco, S. Falconi, W.A. Crichton and M. Mezouar, Phys. Rev. Lett. 90, 255701 (2003).

[19] D.J. Lacks, Phys. Rev. Lett. 84, 4629 (2000).

[20] P. T. Cummings and G. Stell, Mol. Phys. 43, 1267 (1981).

[21] M. Togaya, Phys. Rev. Lett. 79, 2474 (1997).

[22] P. A. Netz, S. Buldyrev, M. C. Barbosa and H. E. Stanley Phys. Rev. E 73, 061504 (2006).

[23] A. B. de Oliveira, P. A. Netz, T. Colla, and M. C. Barbosa, J. Chem. Phys. 124, 084505 (2006).

[24] J.D. Bernal and R.H. Fowler, J. Chem. Phys. 1, 515 (1933).

[25] Vera B. Henriques and Marcia C. Barbosa, Phys. Rev. E 71 (2005).

[26] Vera B. Henriques, Nara Guisoni, Marco Aurelio Barbosa, Marcelo Thielo and Marcia C. Barbosa, Molecular Physics 103, 3001 (2005).

[27] K.A.T. Silverstein, A.D.J.Haymet and K.A. Dill, J. Am. Chem. Soc. 120, 3166 (1998).

[28] T.M.Truskett, P.G. Debenedetti, S. Sastry and S. Torquato, J. Chem. Phys. 111, 2647 (1999).

[29] T.M.Truskett and K.A. Dill, J. Chem. Phys. 117, 5101 (2002).

[30] P. Kumar, S. V. Buldyrev, F. Sciortino, E. Zaccarelli and H. E. Stanley, Phys. Rev. E 72, 021501 (2005). 
[31] G. Franzese, M.I. Marques and H.E. Stanley, Phys Rev E 67, 011103 (2003).

[32] E. A. Jagla, Phys. Rev. E 58, 1478 (1998); E. A. Jagla, J. Chem. Phys. 110, 451 (1999); E. A. Jagla, J. Chem. Phys. 111, 8980 (1999); E. A. Jagla, Phys. Rev. E 63, 061501 (2001); E. A. Jagla, Phys. Rev. E 63, 061509 (2001).

[33] N. B. Wilding and J. E. Magee, Phys. Rev. E 66, 031509 (2002).

[34] P. J. Camp, Phys. Rev. E. 68, 061506 (2003).

[35] P. J. Camp, Phys. Rev. E. 71, 031507 (2005). 